\title{
Stabilized Heterosis Studies for Seed Cotton Yield and Component Traits in Upland Cotton (Gossypium hirsutum L.)
}

\author{
Harish Bhagwan Kumbhalkar ${ }^{1 *}$, Vijaykumar Lachapatrao Gawande ${ }^{2}$, \\ Surendra Bhimrao Deshmukh ${ }^{3}$, Vinita Prashant Gotmare ${ }^{1}$ \\ and Vijay Namdeo Waghmare ${ }^{1}$
}

${ }^{1}$ Division of Crop Improvement, ICAR-Central Institute for Cotton Research, Post Bag No. 2, Shankar Nagar P.O. Nagpur-440010, Maharashrra, India

${ }^{2}$ Pulses Research Unit, ${ }^{3}$ Cotton Research Unit, Dr. Panjabrao Deshmukh Krishi Vidyapeeth, Akola-444104, Maharashtra, India

*Corresponding author

\section{A B S T R A C T}

The study was undertaken to evaluate the magnitude of heterosis in 36 cotton hybrids developed by crossing 3 lines and 12 testers in $\mathrm{L} \times \mathrm{T}$ design at three

Keywords

Gossypium

hirsutum (L.), line $\mathrm{x}$ tester, seed cotton yield, standard heterosis

Article Info

Accepted: 12 June 2021 Available Online: 10 July 2021 different locations of Maharashtra State viz., Akola, Amravati and Nagpur during kharif, 2017-18. The cross AKH 8828 x AKH 9916 exhibited highest and significant heterosis over the check PKV Hy-2 for seed cotton yield/plant (60.30 $\%)$, number of bolls/plant $(23.55 \%)$ and fibre strength $(10.43 \%)$ across the environments. The cross AKH 081 x AKH 09-5 exhibited higher and significant standard heterosis $(54.05 \%)$ for seed cotton yield/plant in association with other economically important traits. Similarly, other crosses viz., AKH 84635 x SURAJ (47.76 \%), AKH $8828 \times$ AKH 2006-2 (47.12\%) and AKH 8828 x DHY 286 $(45.79 \%)$ also exhibited higher significant heterosis over the check hybrid PKV Hy-2 for seed cotton yield/plant in association with other yield contributing characters. The cross AKH 84635 x AKH 2012-8 exhibited considerable heterosis for seed cotton yield/plant and fibre quality parameters. These promising crosses have immense value to exploitation at commercial level after thorough multilocation testing.

\section{Introduction}

Cotton (Gossypium hirsutum L.) is the king of fibre and an important cash crop of India which exercise profound influence on economics and social affairs. Cotton is the most important raw material for Indian textile industry, which makes up 70 per cent of its raw material needs. In cotton, now the yield levels have reached a plateau. So, the 
increased productivity of cotton could be achieved by selection of parents on the basis of combining ability effects and exploitation of hybrid vigour adopting promising high yielding hybrid cotton cultivars that has been a line of approach in cotton improvement since after release of first cotton hybrid $\mathrm{H} 4$ in India (Patel, 1971 ). Application of biometrical techniques like line $\mathrm{x}$ tester analysis has appeared to be the best and vastly useful breeding tool, which gives generalized picture of genetics of the characters under study. The study of heterosis has a direct bearing on the breeding methodology to be employed for hybrid/varietal improvement and also provides useful information about usefulness of the parents in breeding programs. The commercial usefulness of the hybrid primarily depends on its performance in comparison with the best commercial variety of the region concerned. Verma and Kumar (1974) and Joshi (1979) emphasized that the success of development of superior varieties and/or hybrids depend on the choice of parents for hybridization. Exploitation of heterosis on commercial scale has revolutionized the production in cotton. Hence, the present investigation was undertaken to measure the extent of heterosis over standard check (PKV HY 2) for seed cotton yield and component traits to identify the best suitable cross combinations which can be utilized for exploitation of heterosis in upland cotton.

\section{Materials and Methods}

In the present study, three lines viz., AKH 84635, AKH 8828 and AKH 081 were crossed with twelve testers viz., AKH 10-2, AKH 105, AKH 10-10, AKH 11-7, AKH 2006-2, AKH 2012-8, AKH 2012-9, AKH 09-5, AKH 976, AKH 9916, DHY 286 and SURAJ in a line $\mathrm{x}$ tester $\mathrm{m}$ a $\mathrm{t}$ i $\mathrm{n} \mathrm{g}$ design (Kempthorne, 1957) to develop 36 hybrids during kharif, 2016 at Cotton Research Unit, Dr. PDKV, Akola. The 15 parents and the 36 hybrids along with 1 check (PKV Hy 2) were evaluated to study the extent of heterosis in randomized block design with three replications across 3 environments at Cotton Research Unit, Dr. PDKV, Akola (lat. $20.41^{0} \mathrm{~N}$ and Long. $\left.77.3^{\circ} \mathrm{E}\right), \quad$ Regional Research Centre, Amravati (lat. $20.96^{\circ} \mathrm{N}$ and long. $77.85^{\circ} \mathrm{E}$ ) and ICAR-Central Institute for Cotton Research, Nagpur (lat. $23.03^{\circ} \mathrm{N}$ and long. $72.40^{\circ} \mathrm{E}$ ) during kharif, 2017-18. The spacing of $60 \times 60 \mathrm{~cm}$ for hybrids and parents was adopted at all the three environments. The observations in each genotype per replication were recorded for number of sympodia per plant, number of bolls per plant, boll weight $(\mathrm{g})$, seed index $(\mathrm{g})$, ginning percentage $(\%)$, seed cotton yield per plant $(\mathrm{g})$, lint index $(\mathrm{g})$, $2.5 \%$ span length $(\mathrm{mm})$, fibre strength $(\mathrm{g} / \mathrm{tex})$, fibre fineness $(\mu \mathrm{g} / \mathrm{inch})$, uniformity index $(\%)$ and elongation percentage (\%). The relative or average heterosis, heterobeltiosis and standard heterosis were calculated as per cent deviation of mean of the $F_{1}$ hybrid from the mid parent (Briggle, 1963), better parent (Bitzer et al., 1968) and standard parental value (Meredith and Bridge, 1972), respectively. The data was analyzed to find out the extent of heterosis according to the model given by Kempthorne, 1957).

\section{Results and Discussion}

Pooled analysis of variance over the three environments revealed that the source of variation due to environment showed highly significant differences for all the characters indicating the validity of conduct of experiment in these environments (Table 1). The parents showed highly significant differences for all the characters except number of sympodia/plant. The variances due to crosses were found highly significant for all the characters. There was a highly significant difference among parents and hybrids for most of the characters under study except number of sympodia/plant, lint index and elongation 
percentage which indicated the presence of variability in the parental lines selected for the study. The average heterosis $\left(\mathrm{H}_{1}\right)$, heterobeltiosis $\left(\mathrm{H}_{2}\right)$ and standard heterosis $\left(\mathrm{H}_{3}\right)$ over the three environments were studied for 12 characters. The extent of heterosis was studied for all the traits against the standard check hybrid PKV Hy 2. The values for the standard heterosis for individual crosses recorded for different characters (Table 2). The range of standard heterosis for seed cotton yield/plant was from 20.94 to 60.30 per cent. Seventeen hybrids recorded significant superior performance over the check for this trait. The important crosses which showed highest heterotic effect were AKH 8828 x AKH 9916 (60.30\%) followed by AKH 081 x AKH 09-5 (54.05\%), AKH 84635 x SURAJ (47.76\%), AKH 8828 x AKH 2006-2 (47.12 $\%$ ) and AKH $8828 \times$ DHY 286 (45.79\%). These hybrids need special attention to exploit heterosis for seed cotton yield. Several earlier workers viz., Sanjay et al., (2010), Patil et al., (2012), Monicashree et al., $\left(2017^{\mathrm{b}}\right)$, Chinchane et al., (2018) and Bilwal et al., (2018) also reported the heterosis for this trait in American cotton.

Among the other characters, the standard heterosis for number of sympodia/plant ranged from -13.78 to 9.61 per cent over the check PKV Hy 2. Only one hybrid AKH 081 x AKH 10-10 (9.61\%) showed significant positive heterosis for number of sympodia/plant over the check hybrid.

The standard heterosis for number of bolls/plant was ranged from 18.38 to 36.99 per cent. Eleven hybrids recorded significant positive superior performance over the check.

The cross AKH $8828 \times$ AKH 2006-2 (36.99 $\%)$ exhibited maximum heterosis over check for number of bolls/plant followed by $\mathrm{AKH}$ 8828 x DHY 286 (36.08\%) and AKH $081 \times$ DHY 286 (27.99\%). Out of 36 hybrids, only one hybrid AKH $081 \times$ AKH 09-5 (13.60\%) exhibited significant and positive heterosis for boll weight over the check PKV Hy-2. Twelve hybrids showed significant superiority over the check PKV Hy-2 for seed index.

The hybrid AKH 081 x AKH 09-5 (24.03\%) followed by AKH 84635 x SURAJ (19.09\%) and AKH 081 x DHY 286 (17.0\%) showed considerable amount of standard heterosis for seed index. Ten hybrids exhibited significant and positive standard heterosis for ginning percentage to the tune of -15.67 to 7.59 per cent. The hybrid AKH 84635 x AKH 10-2 $(7.59 \%)$ showed highest significant heterosis followed by AKH 84635 x AKH 2012-8 (6.82 $\%)$ and AKH $081 \times$ AKH 10-10 (5.95\%). Fourteen hybrids showed significant positive heterosis for lint index over the check PKV Hy 2. The cross AKH 84635 x AKH 2012-8 (26.0\%) exhibited highest positive standard heterosis followed by crosses AKH $081 \mathrm{x}$ AKH 10-10 (25.47\%) and AKH $84635 \mathrm{x}$ AKH 10-2 (23.05\%) for lint index.

Total 18 hybrids showed significant superiority over the check PKV Hy-2 for fibre quality trait. The hybrid AKH 081 x AKH 10$10(7.35 \%)$ ranked first for standard heterosis followed by $\mathrm{AKH} 84635$ x AKH 09-5 (7.27 $\%)$ and AKH 081 x SURAJ (6.09\%) for 2.5 $\%$ span length.

The cross AKH $081 \times$ AKH 10-10 (15.88 \%) showed maximum heterosis for fibre strength over check. The negative heterosis is desirable for fibre fineness. The cross AKH $84635 \mathrm{x}$ AKH 09-5 and AKH 081 x AKH 976 (-11.11 $\%)$ showed highest negative heterosis over standard check followed by AKH 081 x AKH 2012-9 (-10.08\%) and AKH 8828 x SURAJ ($9.04 \%)$. The cross AKH $8828 \times$ AKH 2006-2 $(19.81 \%)$ exhibited highest heterosis for elongation percentage followed by the crosses AKH 8828 x DHY 286 (12.04 \%) and AKH $081 \times$ AKH 10-10 (11.26\%). 
Table.1 Pooled analysis of variance for experimental design over the three environments for various traits in cotton

\begin{tabular}{|c|c|c|c|c|c|c|c|c|c|c|c|c|c|}
\hline \multirow{2}{*}{$\begin{array}{c}\text { Sources of } \\
\text { variation }\end{array}$} & \multirow[t]{2}{*}{ d.f. } & & & & & & & \multicolumn{6}{|c|}{ Mean sum of squares } \\
\hline & & $\begin{array}{l}\text { Number } \\
\text { of } \\
\text { sympodia }\end{array}$ & $\begin{array}{l}\text { Number of } \\
\text { bolls/plant }\end{array}$ & $\begin{array}{c}\text { Boll } \\
\text { weight }\end{array}$ & $\begin{array}{l}\text { Seed } \\
\text { index }\end{array}$ & $\begin{array}{c}\text { Ginning } \\
\text { percentage }\end{array}$ & $\begin{array}{l}\text { Seed cotton } \\
\text { yield/plant }\end{array}$ & $\begin{array}{l}\text { Lint } \\
\text { index }\end{array}$ & $\begin{array}{l}2.5 \% \\
\text { Span } \\
\text { length }\end{array}$ & $\begin{array}{l}\text { Fibre } \\
\text { strength }\end{array}$ & $\begin{array}{c}\text { Fibre } \\
\text { fineness }\end{array}$ & $\begin{array}{l}\text { Uniformity } \\
\text { index }\end{array}$ & $\begin{array}{l}\text { Elongation } \\
\text { percentage }\end{array}$ \\
\hline Environments & 2 & $255.14 * *$ & $33217.83 * *$ & $9.47 * *$ & $25.36 * *$ & $204.60 * *$ & $334810.34 * *$ & $4.25 * *$ & $90.57 * *$ & $892.63 * *$ & $50.62 * *$ & $118.44 * *$ & $24.74 * *$ \\
\hline Genotypes & 50 & $6.28 * *$ & $127.87 * *$ & $0.74 * *$ & $3.44 * *$ & $41.23 * *$ & $1906.78 * *$ & $2.44 * *$ & $12.41 * *$ & $20.20 * *$ & $0.99 * *$ & $10.54 * *$ & $1.22 * *$ \\
\hline Parents & 14 & 2.09 & $58.28 * *$ & $0.71 * *$ & $3.42 * *$ & $60.23 * *$ & $796.64 * *$ & $2.92 * *$ & $12.63 * *$ & $14.06 * *$ & $1.31 * *$ & $15.22 * *$ & $2.22 * *$ \\
\hline Crosses & 35 & $7.99 * *$ & $118.59 * *$ & $0.75 * *$ & $3.33 * *$ & $32.23 * *$ & $1749.83 * *$ & $2.32 * *$ & $11.73 * *$ & $20.65 * *$ & $0.63 * *$ & $7.44 * *$ & $0.85 * *$ \\
\hline $\begin{array}{c}\text { Parents Vs } \\
\text { crosses }\end{array}$ & 1 & 5.07 & $1427.03 * *$ & $0.68 * *$ & $7.33 * *$ & $90.07 * *$ & $22941.98 * *$ & 0.06 & $33.06 * *$ & $90.49 * *$ & $9.16 * *$ & $53.25 * *$ & 0.02 \\
\hline $\begin{array}{c}\text { Environments } \\
\text { x genotypes }\end{array}$ & 100 & $4.79 * *$ & $63.56 * *$ & $0.40 * *$ & $1.20 * *$ & $10.04 * *$ & $1107.42 * *$ & $0.54 * *$ & $4.91 * *$ & $9.69 * *$ & $0.55 * *$ & $5.93 * *$ & $0.81 * *$ \\
\hline $\begin{array}{c}\text { Environments } \\
\text { x parents }\end{array}$ & 28 & $5.00 * *$ & $47.30 * *$ & $0.55^{* *}$ & $1.17 * *$ & $7.91 * *$ & $797.85 * *$ & $0.46^{* *}$ & $4.12 * *$ & $12.68 * *$ & $0.67 * *$ & $7.03 * *$ & $0.59 * *$ \\
\hline $\begin{array}{l}\text { Environments } \\
\text { x crosses }\end{array}$ & 70 & $4.60 * *$ & $70.34 * *$ & $0.34 * *$ & $1.20 * *$ & $10.75^{* *}$ & $1187.45^{* *}$ & $0.57 * *$ & $4.84 * *$ & $7.40 * *$ & $0.49 * *$ & $5.15^{* *}$ & $0.90 * *$ \\
\hline $\begin{array}{c}\text { Environments } \\
\text { x parent Vs } \\
\text { crosses }\end{array}$ & 2 & $8.56^{*}$ & 53.84 & 0.23 & $1.19 *$ & $14.73 * *$ & $2640.26^{* *}$ & $0.54 *$ & $18.10 * *$ & $47.99 * *$ & $1.20 * *$ & $17.60 * *$ & $0.79 * *$ \\
\hline Error & 300 & 2.62 & 23.29 & 0.08 & 0.26 & 1.75 & 216.79 & 0.12 & 0.1 & 0.24 & 0.01 & 0.76 & 0.01 \\
\hline
\end{tabular}

*-Significant at $5 \%$ level of significance

**-Significant at $1 \%$ level of significance 
Table.2 Average heterosis $\left(\mathrm{H}_{1}\right)$, heterobeltiosis $\left(\mathrm{H}_{2}\right)$ and standard heterosis $\left(\mathrm{H}_{3}\right)(\%)$ estimated over the three environments for different quantitative traits

\begin{tabular}{|c|c|c|c|c|c|c|c|c|c|c|}
\hline \multirow{2}{*}{$\begin{array}{l}\text { Sr. } \\
\text { No. }\end{array}$} & \multirow[t]{2}{*}{ Crosses } & \multicolumn{3}{|c|}{ Number of sympodia } & \multicolumn{3}{|c|}{ Number of bolls/plant } & \multicolumn{3}{|c|}{ Boll weight } \\
\hline & & $\mathrm{H}_{1}$ & $\mathrm{H}_{2}$ & $\mathrm{H}_{3}$ & $\mathrm{H}_{1}$ & $\mathrm{H}_{2}$ & $\mathrm{H}_{3}$ & $\mathrm{H}_{1}$ & $\mathrm{H}_{2}$ & $\mathrm{H}_{3}$ \\
\hline 1 & $\begin{array}{l}\text { AKH } 84635 \text { X AKH } 10- \\
2\end{array}$ & 4.86 & 2.09 & 1.78 & $26.18 * *$ & $17.60 *$ & 15.23 & $9.40 *$ & 7.76 & -6.3 \\
\hline 2 & $\begin{array}{l}\text { AKH } 84635 \text { X AKH } 10- \\
5\end{array}$ & $-12.44 * *$ & $-13.51 * *$ & $-13.78 * *$ & 5.97 & 3.26 & 1.18 & -1.58 & $-9.58 *$ & $-8.94 *$ \\
\hline 3 & $\begin{array}{c}\text { AKH } 84635 \text { X AKH } 10- \\
10\end{array}$ & 3.24 & 2.8 & 3.37 & 7.08 & 6.05 & 3.91 & -3.21 & $-10.23 * *$ & $-11.44 * *$ \\
\hline 4 & $\begin{array}{c}\text { AKH } 84635 \text { X AKH } 11- \\
7\end{array}$ & 4.37 & 4.05 & 3.74 & $24.28 * *$ & $17.83^{*}$ & 15.46 & $10.37 * *$ & 3.94 & -0.77 \\
\hline 5 & $\begin{array}{c}\text { AKH } 84635 \text { X AKH } \\
2006-2\end{array}$ & $-13.07 * *$ & $-13.55 * *$ & $-12.86 * *$ & 2.27 & 1.45 & 1.03 & 5.64 & 4.55 & $-9.96 *$ \\
\hline 6 & $\begin{array}{c}\text { AKH } 84635 \text { X AKH } \\
2012-8\end{array}$ & 5.11 & 2.95 & 2.63 & 14.07 & 11.94 & 13.94 & $14.03 * *$ & 7.73 & 2.15 \\
\hline 7 & $\begin{array}{c}\text { AKH } 84635 \text { X AKH } \\
2012-9\end{array}$ & 3.6 & 0.86 & 0.55 & $18.22 *$ & 13.91 & $20.39 *$ & 4.05 & 1.19 & $-9.68 *$ \\
\hline 8 & $\begin{array}{l}\text { AKH } 84635 \text { X AKH 09- } \\
5\end{array}$ & -3.43 & -4.91 & -5.21 & -3.69 & -6.4 & -8.28 & -6.06 & $-17.96 * *$ & -7.33 \\
\hline 9 & $\begin{array}{c}\text { AKH } 84635 \text { X AKH } \\
976\end{array}$ & -1.24 & -2.46 & -2.76 & 10.45 & 8.57 & 6.38 & 3.25 & -2.82 & -7.1 \\
\hline 10 & $\begin{array}{c}\text { AKH } 84635 \text { X AKH } \\
9916\end{array}$ & 3.62 & 1.97 & 1.65 & 1.73 & -6.44 & 9.23 & -6.65 & -7.65 & $-20.41 * *$ \\
\hline 11 & $\begin{array}{c}\text { AKH } 84635 \text { X DHY } \\
286\end{array}$ & 5.65 & 4.44 & 6.55 & $26.68 * *$ & $20.81 *$ & $18.38 *$ & 6.13 & -0.3 & -4.31 \\
\hline 12 & AKH 84635 X SURAJ & 0.76 & -2.58 & -2.88 & $25.37 * *$ & $23.72 * *$ & $21.23 *$ & $9.08^{*}$ & -1.65 & 3.28 \\
\hline 13 & AKH 8828 X AKH 10-2 & 0.97 & 0.26 & -3.98 & -6.17 & $-16.27 *$ & -9.68 & $-20.15^{* *}$ & $-26.90 * *$ & $-23.50 * *$ \\
\hline 14 & AKH 8828 X AKH 10-5 & 1.4 & 0.63 & -2.14 & 1.1 & -5.88 & 1.52 & $-14.92 * *$ & $-16.52 * *$ & $-12.63 * *$ \\
\hline 15 & $\begin{array}{c}\text { AKH } 8828 \text { X AKH 10- } \\
10\end{array}$ & $7.42 *$ & 4.87 & 5.45 & $19.07 * *$ & 12.57 & $21.42 *$ & $-6.47 *$ & $-9.15^{*}$ & -4.92 \\
\hline 16 & AKH 8828 X AKH 11-7 & -3.21 & -4.82 & -5.7 & 10.09 & -0.14 & 7.71 & -4.79 & $-8.97 *$ & -4.73 \\
\hline
\end{tabular}




\begin{tabular}{|c|c|c|c|c|c|c|c|c|c|c|}
\hline 17 & $\begin{array}{c}\text { AKH } 8828 \text { X AKH } \\
2006-2\end{array}$ & 2.06 & -0.49 & 0.31 & $32.08 * *$ & $27.01 * *$ & $36.99 * *$ & $11.10 * *$ & 1.26 & 5.98 \\
\hline 18 & $\begin{array}{c}\text { AKH } 8828 \text { X AKH } \\
2012-8\end{array}$ & -2.59 & -2.69 & -6.8 & 4.6 & 1.65 & 9.65 & $-12.89 * *$ & $-16.98 * *$ & $-13.11 * *$ \\
\hline 19 & $\begin{array}{c}\text { AKH } 8828 \text { X AKH } \\
2012-9\end{array}$ & 7.02 & 6.27 & 1.78 & 10.9 & 9.79 & $18.42 *$ & -1.67 & $-8.91 *$ & -4.66 \\
\hline 20 & AKH 8828 X AKH 09-5 & 0.06 & -0.38 & -3.74 & 12.68 & 4.65 & 12.88 & -5.73 & $-9.19 * *$ & 2.57 \\
\hline 21 & AKH 8828 X AKH 976 & 4.82 & 4.03 & 1.16 & $17.82 *$ & 10.6 & $19.29 *$ & 6.26 & 1.66 & 6.4 \\
\hline 22 & $\begin{array}{c}\text { AKH } 8828 \text { X AKH } \\
9916\end{array}$ & 5.48 & 5.08 & 1.41 & 10.01 & 5.82 & $23.55 * *$ & -4.97 & $-13.36 * *$ & $-9.32 *$ \\
\hline 23 & AKH 8828 X DHY 286 & 6.13 & 2.88 & 4.96 & $38.31 * *$ & $26.16 * *$ & $36.08 * *$ & $-10.45 * *$ & $-14.16 * *$ & $-10.16 * *$ \\
\hline 24 & AKH 8828 X SURAJ & 7.13 & 5.63 & 1.16 & 4.52 & -1.51 & 6.23 & $-13.97 * *$ & $-14.11 * *$ & $-9.80 *$ \\
\hline 25 & AKH 081 X AKH 10-2 & 0.32 & -0.13 & -4.84 & $24.26 * *$ & $22.56^{*}$ & 6.68 & 2.24 & -2.26 & -6.81 \\
\hline 26 & AKH 081 X AKH 10-5 & 1.15 & 0.13 & -2.63 & 15.32 & 11.64 & 3.8 & 4.12 & 1.34 & 2.06 \\
\hline 27 & AKH 081 X AKH 10-10 & $11.94 * *$ & $9.01 *$ & $9.61 *$ & 5.02 & 0.08 & -3.84 & -3.73 & -5.34 & -6.62 \\
\hline 28 & АКН 081 X АKH 11-7 & -5.48 & -7.29 & $-8.14 *$ & 8.6 & 8.13 & -5.05 & -6.27 & -6.33 & $-10.58 * *$ \\
\hline 29 & $\begin{array}{c}\text { AKH } 081 \text { X AKH 2006- } \\
2\end{array}$ & -6.43 & $-8.99 *$ & $-8.27 *$ & 0.37 & -5.95 & -6.34 & -2.71 & -7.42 & $-11.73 * *$ \\
\hline 30 & $\begin{array}{c}\text { AKH } 081 \text { X AKH 2012- } \\
8\end{array}$ & -1.19 & -1.35 & -5.7 & 2.33 & -5.07 & -3.38 & 4.36 & 4.08 & -0.77 \\
\hline 31 & $\begin{array}{l}\text { AKH } 081 \text { X AKH 2012- } \\
9\end{array}$ & 1.48 & 1.03 & -3.74 & -6.52 & -14.77 & -9.91 & $-13.01 * *$ & $-15.78 * *$ & $-19.70 * *$ \\
\hline 32 & АКН 081 Х АКН 09-5 & 2.11 & 1.39 & -2.02 & $28.96 * *$ & $25.17 * *$ & 15.76 & $9.07 * *$ & 0.57 & $13.60 * *$ \\
\hline 33 & AKH 081 X АKH 976 & $7.89 *$ & 6.8 & 3.86 & $38.25 * *$ & $32.70 * *$ & $25.60 * *$ & $-20.30 * *$ & $-20.41 * *$ & $-23.92 * *$ \\
\hline 34 & АКН 081 X АКН 9916 & -0.77 & -1.4 & -4.84 & 2.83 & -10.25 & 4.79 & -2.85 & -7.52 & $-11.83 * *$ \\
\hline 35 & AKH 081 X DHY 286 & -3.54 & -6.72 & -4.84 & $45.48 * *$ & $43.96 * *$ & $27.99 * *$ & $-11.93 * *$ & $-12.22 * *$ & $-15.75 * *$ \\
\hline 36 & AKH 081 X SURAJ & 6.89 & 5.66 & 0.67 & 6.54 & 1.87 & -2.81 & -0.43 & -5.02 & -0.26 \\
\hline & $\mathrm{SE}(\mathrm{m}) \pm$ & 0.64 & 0.74 & 0.74 & 2.11 & 2.43 & 2.43 & 0.12 & 0.13 & 0.13 \\
\hline & $\mathrm{CD}(5 \%)$ & 1.26 & 1.45 & 1.45 & 4.15 & 4.79 & 4.79 & 0.23 & 0.26 & 0.26 \\
\hline & CD (1\%) & 1.66 & 1.92 & 1.92 & 5.48 & 6.32 & 6.32 & 0.30 & 0.35 & 0.35 \\
\hline
\end{tabular}

*- Significant at $5 \%$ level of significance

**-Significant at $1 \%$ level of significance 
Table.2 Cont...

\begin{tabular}{|c|c|c|c|c|c|c|c|c|c|c|}
\hline \multirow{2}{*}{$\begin{array}{l}\text { Sr. } \\
\text { No. }\end{array}$} & \multirow[t]{2}{*}{ Crosses } & \multicolumn{3}{|c|}{ Seed index } & \multicolumn{3}{|c|}{ Ginning percentage } & \multicolumn{3}{|c|}{ Seed cotton yield/plant } \\
\hline & & $\mathrm{H}_{1}$ & $\mathrm{H}_{2}$ & $\mathrm{H}_{3}$ & $\mathrm{H}_{1}$ & $\mathrm{H}_{2}$ & $\mathrm{H}_{3}$ & $\mathrm{H}_{1}$ & $\mathrm{H}_{2}$ & $\mathrm{H}_{3}$ \\
\hline 1 & $\begin{array}{c}\text { AKH } 84635 \text { X AKH 10- } \\
2\end{array}$ & $5.55 *$ & 3.15 & $11.18 * *$ & $12.31 * *$ & $8.93 * *$ & $7.59 * *$ & $20.21 *$ & 14.28 & 4.88 \\
\hline 2 & $\begin{array}{c}\text { AKH } 84635 \text { X AKH 10- } \\
5\end{array}$ & $-5.34 *$ & $-9.50 * *$ & 2.08 & $9.18 * *$ & $8.22 * *$ & 0.47 & 8.67 & 7.13 & 1.19 \\
\hline 3 & $\begin{array}{c}\text { AKH } 84635 \text { X AKH 10- } \\
10\end{array}$ & $-7.30 * *$ & $-15.32 * *$ & 5.36 & $7.76 * *$ & $5.30 * *$ & 2.42 & 4.23 & -3.11 & 3.51 \\
\hline 4 & $\begin{array}{c}\text { AKH } 84635 \text { X AKH } 11- \\
7\end{array}$ & 3.02 & -2.19 & $11.96 * *$ & $6.67 * *$ & 3.4 & 2.26 & $49.14 * *$ & $47.97 * *$ & $37.97 * *$ \\
\hline 5 & $\begin{array}{c}\text { AKH } 84635 \text { X AKH } \\
2006-2\end{array}$ & -3.88 & -5.76 & 0.91 & $9.52 * *$ & 1.95 & $-5.36 * *$ & 0.07 & -0.13 & -8.34 \\
\hline 6 & $\begin{array}{c}\text { AKH } 84635 \text { X AKH } \\
2012-8\end{array}$ & $5.47 *$ & 0.95 & $13.60 * *$ & $13.33 * *$ & $11.64 * *$ & $6.82 * *$ & $32.06 * *$ & 13.83 & $44.32 * *$ \\
\hline 7 & $\begin{array}{c}\text { AKH } 84635 \text { X AKH } \\
2012-9\end{array}$ & -4.07 & -4.26 & -1.49 & $-3.45 *$ & $-7.71 * *$ & $-6.03 * *$ & $49.35 * *$ & $40.13 * *$ & $28.61 * *$ \\
\hline 8 & $\begin{array}{c}\text { AKH } 84635 \text { X AKH 09- } \\
5\end{array}$ & $-8.82 * *$ & $-13.26 * *$ & -1.13 & -1.7 & -2.12 & $-9.14 * *$ & -2.45 & -10.72 & -1.33 \\
\hline 9 & AKH 84635 X AKH 976 & $-7.72 * *$ & $-10.92 * *$ & $-8.35 * *$ & $10.69 * *$ & $9.49 * *$ & 1.64 & $61.55 * *$ & $56.12 * *$ & $43.28 * *$ \\
\hline 10 & $\begin{array}{c}\text { AKH } 84635 \text { X AKH } \\
9916\end{array}$ & 0.95 & -2.13 & 0.7 & $6.46 * *$ & 1.46 & $3.96 *$ & $44.19 * *$ & $32.45 * *$ & $45.20 * *$ \\
\hline 11 & AKH 84635 X DHY 286 & 0.91 & -0.33 & 5.14 & $-8.57 * *$ & $-9.16 * *$ & $-15.67 * *$ & $30.21 * *$ & $28.68 * *$ & $20.94 *$ \\
\hline 12 & AKH 84635 X SURAJ & $7.97 * *$ & 1.17 & $19.09 * *$ & $-3.85 *$ & $-9.87 * *$ & $-4.36 *$ & $57.08 * *$ & $53.35 * *$ & $47.76 * *$ \\
\hline 13 & AKH 8828 X AKH 10-2 & $-6.53 *$ & $-9.16 * *$ & -2.09 & $-8.55 * *$ & $-12.94 * *$ & $-4.88 * *$ & $26.76^{*}$ & $24.85 *$ & 6.48 \\
\hline 14 & AKH 8828 X AKH 10-5 & $-6.56 *$ & $-11.14 * *$ & 0.22 & $-3.52 *$ & $-11.49 * *$ & -3.3 & -0.9 & -5.7 & -10.94 \\
\hline 15 & $\begin{array}{c}\text { AKH } 8828 \text { X AKH 10- } \\
10\end{array}$ & -4.74 & $-13.43 * *$ & $7.71 *$ & $-4.98 * *$ & $-10.20 * *$ & -1.89 & $34.15 * *$ & $20.63 *$ & $28.87 * *$ \\
\hline 16 & AKH 8828 X AKH 11-7 & -0.19 & $-5.74 *$ & $7.90^{*}$ & $-8.26 * *$ & $-12.61 * *$ & $-4.52 *$ & 14.72 & 9.83 & 2.41 \\
\hline 17 & $\begin{array}{c}\text { AKH } 8828 \text { X AKH } \\
2006-2\end{array}$ & 2.72 & 0.15 & $7.24 *$ & $6.78 * *$ & $-7.52 * *$ & 1.04 & $66.51 * *$ & $60.92 * *$ & $47.12 * *$ \\
\hline 18 & AKH 8828 X AKH & $-6.08 *$ & $-10.59 * *$ & 0.61 & 2.2 & $-4.15 *$ & $4.72 * *$ & 9.59 & -8.34 & 16.2 \\
\hline
\end{tabular}




\begin{tabular}{|c|c|c|c|c|c|c|c|c|c|c|}
\hline & $2012-8$ & & & & & & & & & \\
\hline 19 & $\begin{array}{c}\text { AKH } 8828 \text { X AKH } \\
2012-9\end{array}$ & $-7.24 * *$ & $-7.58 *$ & -5.28 & $-4.96 * *$ & $-8.19 * *$ & 0.3 & $53.76 * *$ & $49.39 * *$ & $27.42 * *$ \\
\hline 20 & AKH 8828 X AKH 09-5 & -3.08 & $-8.29 * *$ & 4.53 & $3.10 *$ & $-5.03 * *$ & $3.76^{*}$ & 3.41 & -8.4 & 1.24 \\
\hline 22 & AKH 8828 X AKH 9916 & -0.5 & -3.01 & -1.34 & -1.07 & $-4.14 *$ & $4.73 * *$ & $64.48 * *$ & $46.22 * *$ & $60.30 * *$ \\
\hline 23 & AKH 8828 X DHY 286 & 1.73 & -0.08 & 5.4 & 0.29 & $-7.79 * *$ & 0.74 & $62.65 * *$ & $55.13 * *$ & $45.79 * *$ \\
\hline 24 & AKH 8828 X SURAJ & $-9.52 * *$ & $-15.66 * *$ & -0.73 & $-7.30 * *$ & $-8.64 * *$ & -0.18 & $32.18 * *$ & $24.59 *$ & 20.05 \\
\hline 27 & AKH 081 X AKH 10-10 & 1.4 & $-7.87 * *$ & $14.62 * *$ & $6.70 * *$ & $4.55^{*}$ & $5.95 * *$ & 0.76 & -4.35 & 13.71 \\
\hline 28 & АKH 081 X АKH 11-7 & $-11.17 * *$ & $-16.14 * *$ & -4.01 & $-9.47 * *$ & $-10.56 * *$ & $-9.37 * *$ & 2.05 & -8.96 & 8.24 \\
\hline 29 & $\begin{array}{c}\text { AKH } 081 \text { X AKH 2006- } \\
2\end{array}$ & -3.66 & $-6.10^{*}$ & 0.55 & $11.69 * *$ & -0.07 & 1.26 & -13.2 & $-23.23 * *$ & -8.73 \\
\hline 30 & $\begin{array}{c}\text { AKH } 081 \text { X AKH 2012- } \\
8\end{array}$ & $5.36^{*}$ & 0.27 & $12.83 * *$ & 2.35 & -0.51 & 0.83 & -3.04 & -6.06 & 19.1 \\
\hline 33 & АKH 081 X АKH 976 & $-7.68 * *$ & $-10.35 * *$ & $-8.87 * *$ & 2.55 & -2.78 & -1.47 & $30.70 * *$ & 12.4 & $33.63 * *$ \\
\hline 34 & АКН 081 X АКН 9916 & 1.92 & -0.61 & 1.03 & -1.03 & -1.57 & 0.85 & -15.04 & $-18.35^{*}$ & -2.93 \\
\hline 35 & AKH 081 X DHY 286 & $12.96 * *$ & $10.91 * *$ & $17.00 * *$ & $-7.72 * *$ & $-12.14 * *$ & $-10.96 * *$ & $28.27 * *$ & 14.83 & $36.52 * *$ \\
\hline \multirow[t]{4}{*}{36} & AKH 081 X SURAJ & $-5.52 *$ & $-11.96 * *$ & 3.63 & -0.02 & -2.26 & $3.71 *$ & 5.23 & -4.74 & 13.25 \\
\hline & $\mathrm{SE}(\mathrm{m}) \pm$ & 0.21 & 0.24 & 0.24 & 0.55 & 0.64 & 0.64 & 6.12 & 7.07 & 7.07 \\
\hline & $\mathrm{CD}(5 \%)$ & 0.42 & 0.48 & 0.48 & 1.09 & 1.26 & 1.26 & 12.06 & 13.93 & 13.93 \\
\hline & $\mathrm{CD}(1 \%)$ & 0.55 & 0.63 & 0.63 & 1.44 & 1.66 & 1.66 & 15.91 & 18.37 & 18.37 \\
\hline
\end{tabular}


Table.2 Cont...

\begin{tabular}{|c|c|c|c|c|c|c|c|c|c|c|}
\hline \multirow{2}{*}{$\begin{array}{l}\text { Sr. } \\
\text { No. }\end{array}$} & \multirow[t]{2}{*}{ Crosses } & \multicolumn{3}{|c|}{ Lint index } & \multicolumn{3}{|c|}{$2.5 \%$ span length } & \multicolumn{3}{|c|}{ Fibre strength } \\
\hline & & $\mathrm{H}_{1}$ & $\mathrm{H}_{2}$ & $\mathrm{H}_{3}$ & $\mathrm{H}_{1}$ & $\mathrm{H}_{2}$ & $\mathrm{H}_{3}$ & $\mathrm{H}_{1}$ & $\mathrm{H}_{2}$ & $\mathrm{H}_{3}$ \\
\hline 1 & $\begin{array}{c}\text { AKH } 84635 \text { X AKH 10- } \\
2\end{array}$ & $24.63 * *$ & $16.56^{* *}$ & $23.05 * *$ & $-7.53 * *$ & $-9.25 * *$ & $-2.83 * *$ & $-2.94 * *$ & $-8.11 * *$ & 0.79 \\
\hline 2 & AKH 84635 X AKH 10- & 5.79 & 2.5 & 0.45 & $-6.38 * *$ & $-7.03 * *$ & $-2.79 * *$ & $-1.57 *$ & $-7.01 * *$ & $2.44 * *$ \\
\hline 3 & $\begin{array}{c}\text { AKH } 84635 \text { X AKH 10- } \\
10\end{array}$ & 5.54 & -6.41 & $11.21 * *$ & $-7.43 * *$ & $-9.29 * *$ & $-2.55 * *$ & $-8.87 * *$ & $-14.40 * *$ & $-4.54 * *$ \\
\hline 4 & $\begin{array}{c}\text { AKH } 84635 \text { X AKH 11- } \\
7\end{array}$ & $12.76 * *$ & 2.51 & $15.14 * *$ & $-6.85 * *$ & $-9.37 * *$ & $-1.22 *$ & $3.34 * *$ & $-2.26 * *$ & $7.42 * *$ \\
\hline 5 & $\begin{array}{c}\text { AKH } 84635 \text { X AKH } \\
2006-2\end{array}$ & $9.70 *$ & 1.09 & -7.09 & $-7.67 * *$ & $-7.85 * *$ & $-4.99 * *$ & $-9.92 * *$ & $-15.29 * *$ & $-5.76 * *$ \\
\hline 6 & $\begin{array}{c}\text { AKH } 84635 \text { X AKH } \\
2012-8\end{array}$ & $27.98 * *$ & $19.99 * *$ & $26.00 * *$ & $3.11 * *$ & $1.75 * *$ & $4.91 * *$ & 0.23 & $-6.11 * *$ & $5.32 * *$ \\
\hline 7 & $\begin{array}{c}\text { AKH } 84635 \text { X AKH } \\
2012-9\end{array}$ & $-9.43 * *$ & $-15.18 * *$ & $-10.71 * *$ & $-9.82 * *$ & $-10.14 * *$ & $-6.68 * *$ & -1.25 & $-7.37 * *$ & $3.62 * *$ \\
\hline 8 & AKH 84635 X AKH 09- & $-11.75 * *$ & $-15.68 * *$ & $-14.93 * *$ & $4.72 * *$ & $4.04 * *$ & $7.27 * *$ & $-3.11 * *$ & $-5.80 * *$ & $-2.27 *$ \\
\hline 9 & AKH 84635 X AKH 976 & 6.84 & 1.35 & -6.86 & $0.94 *$ & -0.04 & $3.06 * *$ & $-6.12 * *$ & $-8.43 * *$ & $-5.63 * *$ \\
\hline 10 & $\begin{array}{c}\text { AKH } 84635 \text { X AKH } \\
9916\end{array}$ & $12.71 * *$ & $7.99 *$ & $8.31 *$ & $-3.48 * *$ & $-6.02 * *$ & $-3.10 * *$ & $5.42 * *$ & $3.84 * *$ & $4.89 * *$ \\
\hline 11 & AKH 84635 X DHY 286 & $-11.22 * *$ & $-11.92 * *$ & $-17.75 * *$ & $-9.07 * *$ & $-10.02 * *$ & $-7.23 * *$ & $2.18 * *$ & -1.61 & $4.14 * *$ \\
\hline 12 & AKH 84635 X SURAJ & -0.33 & $-14.73 * *$ & $10.21 * *$ & $-3.90 * *$ & $-6.36 * *$ & $1.77 * *$ & $8.37 * *$ & $2.42 * *$ & $12.74 * *$ \\
\hline 13 & AKH 8828 X AKH 10-2 & $-17.31 * *$ & $-21.29 * *$ & $-8.07 *$ & $-5.19 * *$ & $-10.86 * *$ & $-4.56 * *$ & $-6.85 * *$ & $-9.39 * *$ & -0.61 \\
\hline 14 & AKH 8828 X AKH 10-5 & $-11.50 * *$ & $-18.63 * *$ & -4.96 & $-6.01 * *$ & $-10.64 * *$ & $-6.56 * *$ & $-10.53 * *$ & $-13.15 * *$ & $-4.32 * *$ \\
\hline 15 & $\begin{array}{c}\text { AKH } 8828 \text { X AKH 10- } \\
10\end{array}$ & $-11.02 * *$ & $-11.78 * *$ & 4.83 & $4.50 * *$ & $-1.90 * *$ & $5.38 * *$ & $-10.08 * *$ & $-13.22 * *$ & $-3.23 * *$ \\
\hline 16 & AKH 8828 X AKH 11-7 & $-12.94 * *$ & $-14.61 * *$ & -0.26 & -0.21 & $-6.96 * *$ & $1.41 * *$ & $-2.33 * *$ & $-5.08 * *$ & $4.32 * *$ \\
\hline 17 & $\begin{array}{c}\text { AKH } 8828 \text { X AKH } \\
2006-2\end{array}$ & $11.63 * *$ & $-7.16^{*}$ & $8.44 *$ & $5.88 * *$ & $1.53 * *$ & $4.28 * *$ & $-3.63 * *$ & $-6.90 * *$ & $3.58 * *$ \\
\hline 18 & AKH 8828 X AKH & -2.68 & $-7.59 *$ & $7.94 *$ & 0.04 & $-3.01 * *$ & $-2.63 * *$ & $-5.62 * *$ & $-9.18 * *$ & $1.88 *$ \\
\hline
\end{tabular}




\begin{tabular}{|c|c|c|c|c|c|c|c|c|c|c|}
\hline & $2012-8$ & & & & & & & & & \\
\hline 19 & $\begin{array}{c}\text { AKH } 8828 \text { X AKH } \\
2012-9\end{array}$ & $-14.19 * *$ & $-18.42 * *$ & -4.72 & 0.08 & $-4.54 * *$ & -0.86 & $-12.45^{* *}$ & $-15.64 * *$ & $-5.63 * *$ \\
\hline 20 & AKH 8828 X AKH 09-5 & 1.78 & -5.15 & $10.79 * *$ & $7.24 * *$ & $3.28 * *$ & $5.11 * *$ & 0.65 & 0.63 & $4.41 * *$ \\
\hline 22 & AKH 8828 X AKH 9916 & -2.21 & $-9.12 * *$ & 6.14 & $4.56 * *$ & $2.74 * *$ & 0.35 & $7.89 * *$ & $6.48 * *$ & $10.43^{* *}$ \\
\hline 23 & AKH 8828 X DHY 286 & 1.58 & $-8.60 *$ & 6.75 & $2.13 * *$ & $-1.25^{*}$ & -0.31 & 1.02 & 1.04 & $5.85^{* *}$ \\
\hline 24 & AKH 8828 X SURAJ & $-19.25 * *$ & $-23.14 * *$ & -0.66 & $3.16 * *$ & $-3.69 * *$ & $4.68 * *$ & $-10.00 * *$ & $-12.60 * *$ & $-3.80 * *$ \\
\hline 27 & AKH 081 X AKH 10-10 & $12.76 * *$ & 5.59 & $25.47 * *$ & $4.14 * *$ & -0.07 & $7.35 * *$ & $9.91 * *$ & $3.91 * *$ & $15.88 * *$ \\
\hline 28 & AKH 081 X AKH 11-7 & $-23.44 * *$ & $-26.37 * *$ & $-17.30 * *$ & $-4.07 * *$ & $-8.58 * *$ & -0.35 & $1.92 *$ & $-2.98 * *$ & $6.63 * *$ \\
\hline 29 & $\begin{array}{l}\text { AKH } 081 \text { X AKH 2006- } \\
2\end{array}$ & $12.97 * *$ & -1.32 & 2.35 & $-2.09 * *$ & $-3.98 * *$ & $-1.38 * *$ & $-10.92 * *$ & $-15.69 * *$ & $-6.20 * *$ \\
\hline 30 & $\begin{array}{l}\text { AKH } 081 \text { X AKH 2012- } \\
8\end{array}$ & $7.09 *$ & 6.43 & $11.76 * *$ & $2.45^{* *}$ & $1.60 * *$ & $2.00 * *$ & $5.73 * *$ & -0.31 & $11.82 * *$ \\
\hline 33 & AKH 081 X AKH 976 & -3.77 & $-13.63 * *$ & $-10.42 * *$ & 0.63 & -0.54 & 0.55 & $-3.43 * *$ & $-5.17 * *$ & $-2.27 *$ \\
\hline 34 & AKH 081 X АKH 9916 & 0.44 & -1.22 & 2.45 & $1.74 * *$ & $1.19 *$ & -0.08 & $2.18 * *$ & 1.34 & $2.36^{*}$ \\
\hline 35 & AKH 081 X DHY 286 & 0.4 & -4.6 & -1.05 & $1.77 * *$ & 0.66 & $1.61 * *$ & 0.11 & $-2.97 * *$ & $2.71 * *$ \\
\hline \multirow[t]{4}{*}{36} & AKH 081 X SURAJ & $-6.00 *$ & $-15.28 * *$ & $9.49 *$ & $2.29 * *$ & $-2.39 * *$ & $6.09 * *$ & $-3.21 * *$ & $-7.93 * *$ & 1.35 \\
\hline & $\mathrm{SE}(\mathrm{m}) \pm$ & 0.14 & 0.16 & 0.16 & 0.13 & 0.15 & 0.15 & 0.21 & 0.24 & 0.24 \\
\hline & $\mathrm{CD}(5 \%)$ & 0.28 & 0.32 & 0.32 & 0.25 & 0.29 & 0.29 & 0.40 & 0.47 & 0.47 \\
\hline & CD (1\%) & 0.37 & 0.43 & 0.43 & 0.34 & 0.39 & 0.39 & 0.53 & 0.62 & 0.62 \\
\hline
\end{tabular}


Table.2 Cont...

\begin{tabular}{|c|c|c|c|c|c|c|c|c|c|c|}
\hline \multirow{2}{*}{$\begin{array}{l}\text { Sr. } \\
\text { No. }\end{array}$} & \multirow[t]{2}{*}{ Crosses } & \multicolumn{3}{|c|}{ Fibre fineness } & \multicolumn{3}{|c|}{ Uniformity index } & \multicolumn{3}{|c|}{ Elongation percentage } \\
\hline & & $\mathrm{H}_{1}$ & $\mathrm{H}_{2}$ & $\mathrm{H}_{3}$ & $\mathrm{H}_{1}$ & $\mathrm{H}_{2}$ & $\mathrm{H}_{3}$ & $\mathrm{H}_{1}$ & $\mathrm{H}_{2}$ & $\mathrm{H}_{3}$ \\
\hline 1 & $\begin{array}{c}\text { AKH } 84635 \text { X AKH 10- } \\
2\end{array}$ & $11.95 * *$ & -1.97 & $2.84 *$ & -0.66 & $-2.09 * *$ & $-2.09 * *$ & -0.2 & $-3.14 * *$ & $-4.27 * *$ \\
\hline 2 & AKH 84635 X AKH 10- & $13.05 * *$ & $6.65 * *$ & $11.89 * *$ & 0.2 & $-1.05 *$ & $-1.44 * *$ & $2.87 * *$ & 0.19 & $4.47 * *$ \\
\hline 3 & $\begin{array}{c}\text { AKH } 84635 \text { X AKH 10- } \\
10\end{array}$ & $-6.78 * *$ & $-8.62 * *$ & $-4.13 * *$ & $-1.39 * *$ & $-3.12 * *$ & $-2.48 * *$ & 0.98 & 0.58 & 0.19 \\
\hline 4 & $\begin{array}{c}\text { AKH } 84635 \text { X AKH 11- } \\
7\end{array}$ & $-4.44 * *$ & $-12.56 * *$ & $-8.27 * *$ & $0.93 *$ & -0.13 & -0.92 & $12.16 * *$ & $2.36 *$ & 1.17 \\
\hline 5 & $\begin{array}{c}\text { AKH } 84635 \text { X AKH } \\
2006-2\end{array}$ & $9.23 * *$ & 1.97 & $6.98 * *$ & $-3.60 * *$ & $-6.12 * *$ & $-3.79 * *$ & $-3.13 * *$ & $-6.25 * *$ & -0.97 \\
\hline 6 & $\begin{array}{c}\text { AKH } 84635 \text { X AKH } \\
2012-8\end{array}$ & $-7.15 * *$ & $-8.87 * *$ & $-4.39 * *$ & $1.34 * *$ & 0.93 & $-1.18 *$ & -0.28 & $-6.23 * *$ & $5.24 * *$ \\
\hline 7 & $\begin{array}{c}\text { AKH } 84635 \text { X AKH } \\
2012-9\end{array}$ & $7.69 * *$ & -1.72 & $3.10 *$ & $1.07 *$ & 0.13 & -0.92 & 0.38 & -1.69 & 1.36 \\
\hline 8 & AKH 84635 X AKH 09- & $-3.23 * *$ & $-15.27 * *$ & $-11.11 * *$ & $1.60 * *$ & 0.39 & -0.13 & -0.9 & $-8.62 * *$ & $6.99 * *$ \\
\hline 9 & AKH 84635 X AKH 976 & $6.52 * *$ & $-5.42 * *$ & -0.78 & 0.47 & -0.53 & $-1.44 * *$ & -1 & $-3.14 * *$ & $-4.27 * *$ \\
\hline 10 & $\begin{array}{c}\text { AKH } 84635 \text { X AKH } \\
9916\end{array}$ & $7.42 * *$ & $3.45 * *$ & $8.53 * *$ & 0.2 & 0.3 & $-2.88 * *$ & $-2.54 * *$ & $-6.65 * *$ & 0.78 \\
\hline 11 & AKH 84635 X DHY 286 & $8.75 * *$ & $7.14 * *$ & $12.40 * *$ & $-0.93 *$ & $-1.85 * *$ & $-2.88 * *$ & $4.73 * *$ & 0.91 & $7.57 * *$ \\
\hline 12 & AKH 84635 X SURAJ & $7.18 * *$ & $-4.43 * *$ & 0.26 & $1.12 * *$ & -0.65 & 0.13 & $-1.69 *$ & $-6.08 * *$ & $1.94 *$ \\
\hline 13 & AKH 8828 X AKH 10-2 & $16.79 * *$ & $5.26 * *$ & $3.36 * *$ & $-1.19 * *$ & $-2.61 * *$ & $-2.61 * *$ & -0.1 & $-4.04 * *$ & $-3.11 * *$ \\
\hline 14 & AKH 8828 X AKH 10-5 & $2.16^{*}$ & -0.53 & -2.33 & $-2.46 * *$ & $-3.67 * *$ & $-4.05 * *$ & -0.28 & $-1.86^{*}$ & $2.33 *$ \\
\hline 15 & $\begin{array}{c}\text { AKH } 8828 \text { X AKH 10- } \\
10\end{array}$ & $3.38 * *$ & 2.05 & $2.84 *$ & 0.33 & $-1.43 * *$ & -0.78 & -1.45 & $-2.12 *$ & -1.17 \\
\hline 16 & AKH 8828 X AKH 11-7 & $8.23 * *$ & 2.11 & 0.26 & 0.67 & -0.4 & $-1.18 *$ & $12.98 * *$ & $2.12 *$ & $3.11 * *$ \\
\hline 17 & $\begin{array}{c}\text { AKH } 8828 \text { X AKH } \\
2006-2\end{array}$ & 1.37 & -2.37 & $-4.13 * *$ & $-0.98 *$ & $-3.57 * *$ & $-1.18 *$ & $15.98 * *$ & $13.42 * *$ & $19.81 * *$ \\
\hline 18 & AKH 8828 X AKH & $-3.76 * *$ & $-5.12 * *$ & $-4.13 * *$ & 0.54 & 0.13 & $-1.96 * *$ & $-1.64 *$ & $-6.57 * *$ & $4.85 * *$ \\
\hline
\end{tabular}




\begin{tabular}{|c|c|c|c|c|c|c|c|c|c|c|}
\hline & $2012-8$ & & & & & & & & & \\
\hline 19 & $\begin{array}{c}\text { AKH } 8828 \text { X AKH } \\
2012-9\end{array}$ & 2.1 & $-3.95 * *$ & $-5.68 * *$ & 0.11 & -0.92 & $-1.96 * *$ & -1.24 & $-2.26^{*}$ & 0.78 \\
\hline 20 & AKH 8828 X АKH 09-5 & $14.16 * *$ & $2.89 *$ & 1.03 & 0.53 & -0.66 & $-1.18 *$ & $-11.49 * *$ & $-17.58 * *$ & $-3.50 * *$ \\
\hline 22 & AKH 8828 X AKH 9916 & $3.70 * *$ & $3.16^{*}$ & 1.29 & $1.21 * *$ & $1.21 *$ & $-1.70 * *$ & 1.12 & $-2.16^{*}$ & $5.63 * *$ \\
\hline 23 & AKH 8828 X DHY 286 & -0.52 & -2.28 & -0.52 & -0.8 & $-1.72 * *$ & $-2.75 * *$ & $7.95 * *$ & $5.10 * *$ & $12.04 * *$ \\
\hline 24 & AKH 8828 X SURAJ & 0.86 & $-7.37 * *$ & $-9.04 * *$ & $-1.26 * *$ & $-2.99 * *$ & $-2.35 * *$ & $-3.24 * *$ & $-6.62 * *$ & 1.36 \\
\hline 27 & AKH 081 X AKH 10-10 & -0.8 & $-4.62 * *$ & $-3.88 * *$ & 0.46 & $-1.04 *$ & -0.39 & $13.47 * *$ & $11.70 * *$ & $11.26^{* *}$ \\
\hline 28 & AKH 081 X AKH 11-7 & $2.44^{*}$ & -0.83 & $-7.75 * *$ & -0.13 & -0.92 & $-1.70 * *$ & $15.81 * *$ & $6.84 * *$ & $3.11 * *$ \\
\hline 29 & $\begin{array}{l}\text { AKH } 081 \text { X AKH 2006- } \\
2\end{array}$ & $15.17 * *$ & $13.89 * *$ & $5.94 * *$ & $-3.85^{* *}$ & $-6.12 * *$ & $-3.79 * *$ & -0.29 & $-4.60 * *$ & 0.78 \\
\hline 30 & $\begin{array}{l}\text { AKH } 081 \text { X AKH 2012- } \\
8\end{array}$ & $-2.80 * *$ & $-6.65 * *$ & $-5.68 * *$ & $0.94 *$ & 0.8 & $-1.31 * *$ & $-7.53 * *$ & $-14.01 * *$ & $-3.50 * *$ \\
\hline 33 & AKH 081 X AKH 976 & 1.93 & $-4.44 * *$ & $-11.11 * *$ & $-1.53 * *$ & $-2.24 * *$ & $-3.14 * *$ & $3.05 * *$ & $2.01 *$ & -1.55 \\
\hline 34 & АКН 081 X АКН 9916 & -1.9 & $-3.99 * *$ & $-6.72 * *$ & 0.11 & -0.27 & $-2.61 * *$ & $-2.18 * *$ & $-7.37 * *$ & 0.12 \\
\hline 35 & AKH 081 X DHY 286 & $10.61 * *$ & $5.84 * *$ & $7.75 * *$ & $-1.73 * *$ & $-2.38 * *$ & $-3.40 * *$ & $-5.93 * *$ & $-10.38 * *$ & $-4.47 * *$ \\
\hline \multirow[t]{4}{*}{36} & AKH 081 X SURAJ & $12.39 * *$ & $5.83 * *$ & -1.55 & $-1.65 * *$ & $-3.12 * *$ & $-2.48 * *$ & $-5.11 * *$ & $-10.38 * *$ & $-2.72 * *$ \\
\hline & $\mathrm{SE}(\mathrm{m}) \pm$ & 0.04 & 0.05 & 0.05 & 0.35 & 0.41 & 0.41 & 0.05 & 0.05 & 0.05 \\
\hline & $\mathrm{CD}(5 \%)$ & 0.09 & 0.10 & 0.10 & 0.70 & 0.81 & 0.81 & 0.09 & 0.11 & 0.11 \\
\hline & CD $(1 \%)$ & 0.12 & 0.13 & 0.13 & 0.92 & 1.06 & 1.06 & 0.12 & 0.14 & 0.14 \\
\hline
\end{tabular}


Table.3 Mean yield performance and standard heterosis $\left(\mathrm{H}_{3}\right)$ of promising crosses over the environments

\begin{tabular}{|c|c|c|c|c|c|}
\hline $\mathbf{S N}$ & Crosses & \multicolumn{2}{|c|}{$\begin{array}{l}\text { Mean yield performance } \\
\text { for Seed cotton yield/plant } \\
(\mathrm{g})\end{array}$} & $\begin{array}{c}\text { Standard } \\
\text { heterosis }\left(\mathrm{H}_{3}\right) \\
\text { for seed cotton } \\
\text { yield }(\%)\end{array}$ & $\begin{array}{c}\text { Significant standard heterosis for other characters in } \\
\text { desirable direction }\end{array}$ \\
\hline 1 & AKH 8828 x AKH 9916 & \multicolumn{2}{|l|}{$110.09 * *$} & $60.30 * *$ & 2,9 \\
\hline 2 & AKH 081 х AKH 09-5 & \multicolumn{2}{|l|}{$105.80 * *$} & $54.05 * *$ & $2,3,4,7$ \\
\hline 3 & AKH 84635 x SURAJ & \multicolumn{2}{|l|}{$101.48 * *$} & $47.76 * *$ & $2,4,7,8,9$ \\
\hline 4 & AKH 8828 x AKH 2006-2 & \multicolumn{2}{|l|}{$101.04 * *$} & $47.12 * *$ & $2,7,8,9,10,12$ \\
\hline 5 & AKH 8828 x DHY 286 & \multicolumn{2}{|l|}{$100.09 * *$} & $45.79 * *$ & $2,9,12$ \\
\hline & \multicolumn{5}{|c|}{ 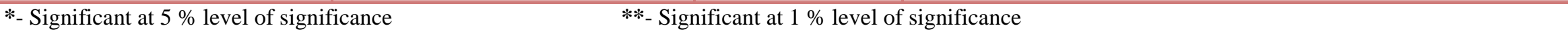 } \\
\hline & 1: Number of sympodia/plant & \multicolumn{4}{|c|}{ 2: Number of bolls/plant 3 : Boll weight } \\
\hline & 4: Seed index & \multirow{2}{*}{\multicolumn{2}{|c|}{$\begin{array}{ll}\text { 5: Ginning percentage } & \text { 6: Seed cotton yield/plant } \\
8: 2.5 \% \text { span length } & 9: \text { Fibre strength }\end{array}$}} & & \\
\hline & 7: Lint index & & \multicolumn{2}{|c|}{ 9: Fibre strength } & \\
\hline & 10: Fibre fineness & 11: Uniformity index & \multicolumn{3}{|c|}{ 12: Elongation percentage } \\
\hline
\end{tabular}


Heterosis for different economically important characters were already reported by Alkuddsi et al., (2013), Chhavikant et al., (2017), Chinchane et al., (2018), Sawarkar et al., (2015), Kannan and Saravanan (2016), Pushpam et al., (2015), Lingaraja et al., (2017) and Karademir and Gencer (2010).

The cross AKH 8828 x AKH 9916 exhibited highest significant heterosis $(60.30 \%)$ over the check PKV Hy-2 for seed cotton yield/plant. This cross also recorded significant standard heterosis in desirable direction for number of bolls/plant and fibre strength over the environments. Similarly, the cross AKH 081 x AKH 09-5 exhibited higher and significant standard heterosis $(54.05 \%)$ for seed cotton yield/plant, number of bolls/plant, boll weight, seed index and lint index over the environments. This cross also exhibited significant heterobeltiosis for important fibre quality traits such as $2.5 \%$ span length and fibre strength. Similarly, other crosses viz., AKH 84635 x SURAJ (47.76 \%), AKH $8828 \times$ AKH 2006-2 (47.12 \%) and AKH 8828 x DHY $286 \quad(45.79 \%)$ also exhibited higher significant heterosis over check hybrid for seed cotton yield/plant and other important yield contributing characters (Table 3). The presence of significant standard heterosis for seed cotton yield, its contributing traits and fibre properties in cotton has been reported by earlier workers viz., Bilwal et al., (2018) and Chinchane et al., (2018). The cross AKH 84635 x AKH 2012-8, though not ranked in top for seed cotton yield/plant based on standard heterotic performance, but, it exhibited satisfactory significant standard heterosis for fibre quality parameters in desirable direction viz., $2.5 \%$ span length, fibre strength, fibre fineness and elongation percentage. Thus, out of 36 hybrids, 3 hybrids viz., AKH $081 \times$ AKH 09-5, AKH $8828 \times$ AKH 2006-2 and AKH $8828 \times$ AKH 9916 were identified as promising over the environments which can be exploited for heterosis breeding programme after multi location testing over the years to confirm their performance.

\section{Acknowledgements}

Sincere thanks to Senior Research Scientist, Cotton Research Unit, Dr. PDKV, Akola, Director, ICAR-Central Institute for Cotton Research, Nagpur and Head, Regional Research Centre, Amravati for providing necessary facilities to conduct research experiment and staff members for help and moral support. This publication is part of Ph.D. research work at Dr. PDKV, Akola.

\section{References}

Alkuddsi Yanal A., Gururaja Rao M. R., Patil, S. S., Gowda, T. H., and Joshi, M. 2013. Combining ability analysis for seed cotton yield (kapas yield) and its components in intra hirsutum hybrids and forming heterotic boxes for exploitation in cotton, Genomics and Applied Biology. 4(5): 35-49.

Bilwal, B. B., Vadodariya, K. V., Lahane, G. R., and Rajkumar, B. K. 2018. Heterosis study for seed cotton yield and its yield attributing traits in upland cotton (Gossypium hirsutum L.) Journal of Pharmacognosy and Phytochemistry, 7(1): 1963-1967.

Bitzer, M. J., Fonesca, S., Papathanasiou, G., and Patterson, F. L. 1968. Coined the term Heterobeltiosis (cited by Fonesca \& Patterson, 1968).

Briggle, L. W. 1963. Heterosis in wheat: A review. Crop Sci., 3: 407-412.

Chhavikant, K. S., Nirania, A. K., and Pundir, S. R. 2017. Heterosis studies for seed cotton yield and other traits in upland cotton (Gossypium hirsutum L.) Journal of Pharmacognosy and Phytochemistry; 6(6): 583-586.

Chinchane, V. N., Duomai, K., and Gopal, G. 
R. 2018. Heterosis studies for qualitative and quantitative characters in cotton (Gossypium arboreum L.) Int.J.Curr.Microbiol.App., Sci, Special Issue-6: 975-982.

Joshi, A. B. 1979. Breeding methodology for autogamous crops. Indian J. Genet., 39(3): 567-578.

Kannan, N., and Saravanan, K. 2016. Heterosis and combining ability analysis in tetraploid Cotton (G.hirsutum and G.barbadense L.). Electronic Journal of Plant Breeding, 7(2): 341-351.

Karademir, E., and Gençer Oktay. 2010. Combining ability and heterosis for yield and fiber quality properties in cotton (G. hirsutum L.) obtained by half diallel mating design. Not. Bot. Hort. Agrobot. Cluj., 38(1): 222-227.

Kempthorne, O. 1957. An introduction to genetic statistics, John Wiley and Son Inc. New york, Champman and Hall Ltd. London, pp., 468-470.

Lingaraja, L., Sangwan, R. S., Nimbal, S., Sangwan, O., and Singh, S. 2017. Heterosis studies for economic and fibre quality traits in line $\mathrm{x}$ tester crosses of upland cotton (Gossypium hirsutum L.). Int. J. Pure App. Biosci., 5(2): 240-248.

Meredith, W. R., and Bridge, R. R. 1972. Heterosis and gene action in cotton, Gossypium hirsutum L. Crop Sci., 12(3): 304-310.
Monicashree, C., Amala Balu P., and Gunasekaran, M. 2017 ${ }^{\mathrm{b}}$. Heterosis studies for yield and fibre quality traits in upland cotton (Gossypium hirsutum L.). Int. J. Pure App. Biosci., 5(3): 169-186.

Patel, C. T. $1971^{\mathrm{b}}$. Hybrid-4, "A new hope towards self sufficiency in cotton in India". Cotton Development (2): p-1.

Patil, S. A., Naik, M. R., Pathak, V. D., and Kumar, V. 2012. Heterosis for yield and fibre properties in upland cotton (Gossypium hirsutum L.) J. Cotton Res. Dev., 26(1) 26-29.

Pushpam, R., Thangaraj, K., and Raveendran, T. S. 2015. Heterosis and combining ability studies in upland cotton for yield characters. Electronic Journal of Plant Breeding, 6(2): 459-463.

Sanjay, B. A., Kajjidoni, S. T., and Talawar, A. M. 2010. Combining ability and heterosis for yield and its components in Interspecific crosses of desi cotton. Agric. Sci. Digest., 30 (3): 157- 162.

Sawarkar, M., Solanke, A., Mhasal, G. S., and Deshmukh, S. B. 2015. Combining ability and heterosis for seed cotton yield, its components and quality traits in Gossypium hirsutum L. Indian Journal of Agricultural Research, 49(2): 154-159.

Verma, M. M., and Kumar, J. 1974. Breeding for yield in self pollinators an introspection and reorientation. Crop Improv., 1 (1 and 2): 15-31.

\section{How to cite this article:}

Harish Bhagwan Kumbhalkar, Vijaykumar Lachapatrao Gawande, Surendra Bhimrao Deshmukh, Vinita Prashant Gotmare and Vijay Namdeo Waghmare. 2021. Stabilized Heterosis Studies for Seed Cotton Yield and Component Traits in Upland Cotton (Gossypium hirsutum L.). Int.J.Curr.Microbiol.App.Sci. 10(07): 99-113. doi: https://doi.org/10.20546/ijcmas.2021.1007.011 